\title{
Alkali promotion of $\mathrm{N}-2$ dissociation over $\mathrm{Ru}(\mathbf{0 0 0 1 )}$
}

\author{
Mortensen, Jens Jørgen; Hammer, Bjørk; Nørskov, Jens Kehlet
}

Published in:

Physical Review Letters

Link to article, DOI:

10.1103/PhysRevLett.80.4333

Publication date:

1998

Document Version

Publisher's PDF, also known as Version of record

Link back to DTU Orbit

Citation (APA):

Mortensen, J. J., Hammer, B., \& Nørskov, J. K. (1998). Alkali promotion of N-2 dissociation over Ru(0001). Physical Review Letters, 80(19), 4333-4339. https://doi.org/10.1103/PhysRevLett.80.4333

\section{General rights}

Copyright and moral rights for the publications made accessible in the public portal are retained by the authors and/or other copyright owners and it is a condition of accessing publications that users recognise and abide by the legal requirements associated with these rights.

- Users may download and print one copy of any publication from the public portal for the purpose of private study or research.

- You may not further distribute the material or use it for any profit-making activity or commercial gain

- You may freely distribute the URL identifying the publication in the public portal

If you believe that this document breaches copyright please contact us providing details, and we will remove access to the work immediately and investigate your claim 


\title{
Alkali Promotion of $\mathbf{N}_{2}$ Dissociation over $\mathrm{Ru}(0001)$
}

\author{
J. J. Mortensen, ${ }^{1}$ B. Hammer, ${ }^{1,2}$ and J. K. Nørskov ${ }^{1}$ \\ ${ }^{1}$ Center for Atomic-scale Materials Physics, Department of Physics, Technical University of Denmark, DK-2800 Lyngby, Denmark \\ ${ }^{2}$ Institute of Physics, Aalborg University, DK-9220 Aalborg, Denmark
}

(Received 12 December 1997)

\begin{abstract}
Using self-consistent density functional calculations, we show that adsorbed $\mathrm{Na}$ and $\mathrm{Cs}$ lower the barrier for dissociation of $\mathrm{N}_{2}$ on $\mathrm{Ru}(0001)$. Since $\mathrm{N}_{2}$ dissociation is a crucial step in the ammonia synthesis reaction, we explain in this way the experimental observation that alkali metals promote the ammonia synthesis reaction over Ru catalysts. We also show that the origin of this effect is predominantly a direct electrostatic attraction between the adsorbed alkali atoms and the dissociating molecule. [S0031-9007(98)06016-5]
\end{abstract}

PACS numbers: 82.65.My, 68.45.Da, 82.65.Jv

Alkali metals are used extensively as promoters of catalytic reactions on metal surfaces. In the ammonia synthesis, for instance, where $\mathrm{N}_{2}$ and $\mathrm{H}_{2}$ are converted into $\mathrm{NH}_{3}$, alkali promoters are used both in the traditional Fe-based catalyst [1] and in the Ru-based catalyst [2,3]. On Fe surfaces, adsorbed potassium is known to increase the rate of $\mathrm{N}_{2}$ dissociation, which is the rate limiting step in the ammonia synthesis, by factors between 8 and 300 depending on the facet $[4,5]$. The effect on the ammonia synthesis rate of adding alkali promoters to $\mathrm{Ru}$ catalysts is as large [6]. Here, the effect has also been shown to depend strongly on the kind of alkali metal added, the trend being that the promotion increases with the atomic number of the alkali metal [6].

There has been a long debate about the origin of the large alkali promotion effect. Coadsorption of alkali metals and other atoms and molecules has been studied extensively on well-defined single crystal surfaces [7]. For $\mathrm{N}_{2}$ dissociation on $\mathrm{Fe}(111)$, preadsorbed $\mathrm{K}$ has been shown to stabilize the adsorbed $\mathrm{N}_{2}$ molecule and to lower the barrier for dissociation [4]. On Ru(0001), on the other hand, preadsorbed $\mathrm{K}$ decreases the stability of adsorbed $\mathrm{N}_{2}$ [8]. On this surface, there is no direct measurement of the effect of alkali atoms on the dissociation, so it is not clear if the promotion of the ammonia synthesis is due to a lowering of the $\mathrm{N}_{2}$ dissociation barrier as for Fe or due to some other effect. There have been several attempts to model the promotional effect of adsorbed alkali metals. One school of thought has focused on the alkali-induced changes in the density of metal states at the Fermi level [9], while others have focused on the electrostatic interaction between the adsorbed alkali atoms and the dissociating molecule [10]. A coherent picture of the relative importance of these effects has not been established yet [11].

In the present Letter, we use self-consistent density functional (DFT) calculations to investigate the effect of adsorbed alkali atoms on the adsorption and dissociation of $\mathrm{N}_{2}$ on $\mathrm{Ru}(0001)$. We show that, while adsorbed alkali atoms destabilize molecularly adsorbed $\mathrm{N}_{2}$ slightly, they lower the barrier for dissociation significantly. We also show that the effect of Cs is considerably larger than that of $\mathrm{Na}$ and investigate the effect of the position of the dissociating molecule relative to the adsorbed $\mathrm{Na}$ or $\mathrm{Cs}$. Using this large database, we can clearly show that the promoting effect is primarily of an electrostatic nature.

In Fig. 1, we summarize the main results of the calculation. The figure shows the calculated energy of the $\mathrm{N}_{2} / \mathrm{Ru}(0001)$ system relative to molecular $\mathrm{N}_{2}$ and the clean $\mathrm{Ru}(0001)$ surface as a function of the dissociation reaction coordinate. The latter is defined as the distance along the minimum energy path connecting the initial, molecularly adsorbed state and the final, dissociated state. Dissociation of $\mathrm{N}_{2}$ on $\mathrm{Ru}(0001)$ is seen to be highly activated. This agrees with the extremely low sticking probability measured for this system [12] and the observation

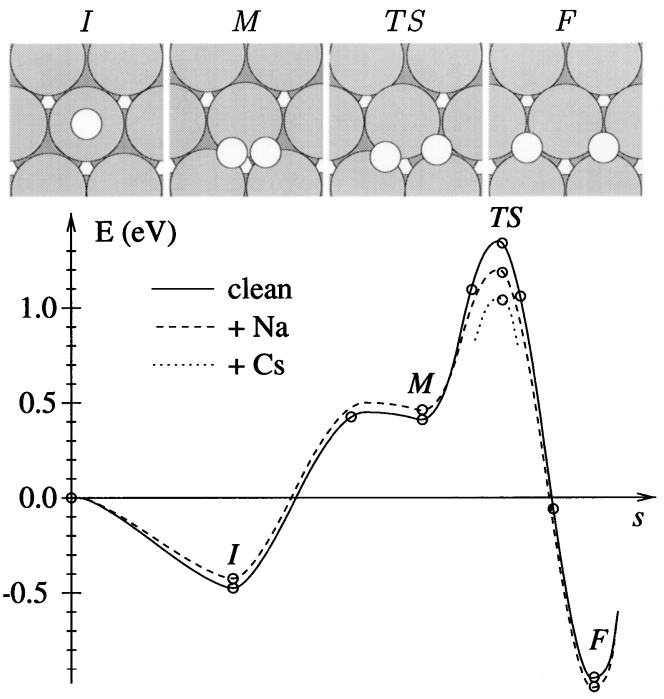

FIG. 1. Top: Snapshots of the minimum energy path for the $\mathrm{N}_{2}$ dissociation reaction: initial state where the molecule is standing perpendicular to the surface $(I)$, metastable state $(M)$, transition state $(T S)$, and final state $(F)$. Bottom: The energy along the path (see text for details). The dashed line shows the energy along the same path in the presence of $\frac{1}{8}$ of a monolayer of $\mathrm{Na}$ (configuration $c$ in Fig. 2). The effect of $\frac{1}{6}$ of a monolayer of $\mathrm{Cs}$ on the transition state is also shown (dotted line, configuration $e$ ). 
in molecular beam scattering experiments that the sticking probability increases by 4 orders of magnitude by increasing the translational energy of the $\mathrm{N}_{2}$ molecules above $1 \mathrm{eV}$ [13]. The figure includes the effect of introducing $\frac{1}{8}$ of a monolayer of $\mathrm{Na}$ in the configuration $c$ shown in Fig. 2. It is seen that the barrier for dissociation is lowered by $0.13 \mathrm{eV}$. The figure also shows that the lowering of the dissociation barrier is even larger $(0.29 \mathrm{eV})$ when $\mathrm{Cs}$ is added to the surface instead of $\mathrm{Na}$. The calculations therefore clearly describe the experimentally observed promoting effect of $\mathrm{Na}$ and $\mathrm{Cs}$ on the ammonia synthesis rate on $\mathrm{Ru}$ surfaces [6], and shows that it can be attributed to a lowering of the activation barrier for $\mathrm{N}_{2}$ dissociation. At the same time, it shows that $\mathrm{Na}$ introduces a slight destabilization of the molecularly adsorbed state, in accordance with the experimental observations for the $\mathrm{K} / \mathrm{N}_{2} / \mathrm{Ru}(0001)$ system [8].

The results are based on self-consistent density functional calculations using the generalized gradient approximation to describe exchange and correlation effects [14]. We use norm conserving pseudopotentials and expand the pseudo-wave-functions in plane waves with a kinetic energy cutoff of 40 Ry [15]. For the Cs pseudopotential, we include $5 p$ semicore states as valence states, and further use the nonlinear core correction scheme, proposed by Louie, Froyen, and Cohen [16]. A Fermi-Dirac function with $k_{B} T=0.05 \mathrm{eV}$ is used to calculate occupation numbers and all energies are extrapolated to $T=0 \mathrm{~K}$. The $\mathrm{Ru}(0001)$ surface is mimicked by a periodic array of two to six layer thick Ru slabs separated by $\sim 9 \AA$ of vacuum. Surface unit cells giving $(2 \times 2),(2 \times 3)$, and $(2 \times 4)$ periodicity along the surface have been used, and the corresponding Brillouin zones have been sampled by 18,12 , and $8 \vec{k}$ points, respectively.

The energy curves in Fig. 1 have been found in the following way. The starting point is a calculation of the minimum energy path and the energy along it for a six layer slab in a $(2 \times 2)$ unit cell. The minimum energy path has been determined by a self-consistent optimization involving all $\mathrm{N}_{2}$ degrees of freedom as well as the $\mathrm{Ru}$ degrees of freedom in the uppermost two layers [17]. This is described in detail in Ref. [18]. This very elaborate calculation is only possible in the relatively small $(2 \times 2)$ unit cell. This cell is so small that interactions between dissociating molecules in adjacent cells cannot be excluded. The cell is also too small for studying the effect of small amounts of adsorbed alkali atoms. We have therefore extended the calculations to the larger $(2 \times 3)$ and $(2 \times 4)$ unit cells by restricting the slab thickness to two layers and by keeping the reaction path determined for the six layer calculation in the small unit cell. We have checked the quality of the two layer slab calculations by comparing to the six layer slab results in a $(2 \times 2)$ unit cell. The error in the absolute binding energies is very small, on the order of $0.1 \mathrm{eV}$. The path also changes only slightly as judged from the small forces on the $\mathrm{N}$ atoms in the two layer calculation. The energy $E(s)$ along the reaction coordinate $s$ for the clean $\mathrm{Ru}(0001)$ surface in Fig. 1 has then been constructed as

$$
E(s)=D_{6}^{(2 \times 2)}(s)+\left[E_{2}^{(2 \times 3)}(s)-E_{2}^{(2 \times 2)}(s)\right],
$$

where $E_{n}^{(k \times l)}$ is the $\mathrm{N}_{2}$-surface interaction energy for an $n$ layer slab with a $(k \times l)$ unit cell. In the same way, the effect of adding, e.g., $\frac{1}{8}$ of a monolayer of $\mathrm{Na}$ is calculated as

$$
E^{\mathrm{Na}}(s)=E(s)+\left[E_{2}^{\mathrm{Na},(2 \times 4)}(s)-E_{2}^{(2 \times 4)}(s)\right],
$$

where the superscript $\mathrm{Na}$ indicates that one $\mathrm{Na}$ has been added per unit cell. The geometry of the different unit cells used is shown in Fig. 2.

The $\mathrm{Na}$ and $\mathrm{Cs}$ atoms have been added in the threefold sites and the equilibrium heights above the Ru surface have been determined to be 2.4 and $3.3 \AA$, respectively. For the $\mathrm{Cs} / \mathrm{Ru}(0001)$ system, where there is an experimental structure determination [19], the agreement with experiment is excellent. By varying the size of the unit cell, we can vary the coverage between $\frac{1}{8}$ for the $(2 \times 4)$ cell and $\frac{1}{4}$ for the $(2 \times 2)$ cell. The calculated changes in work function agree very well with experiment for both adsorbates as seen in Fig. 3.

Having established that the DFT calculations can describe the promoting effect of alkali adsorption on $\mathrm{N}_{2}$ dissociation at least qualitatively, we now turn to the problem of the origin of the effect. We investigate this by looking at the effect of two different alkali atoms, $\mathrm{Na}$ and $\mathrm{Cs}$, and the effect of coverage and geometrical arrangement of the alkali atoms relative to the dissociating molecule. To this end, we concentrate on the alkali-induced change $\Delta E_{\mathrm{TS}}$ in the energy of the transition state, and we keep the geometry of this state fixed as we vary the position and kind of the surrounding alkali atoms. We will return to the role of alkali-induced changes in the reaction path later. The

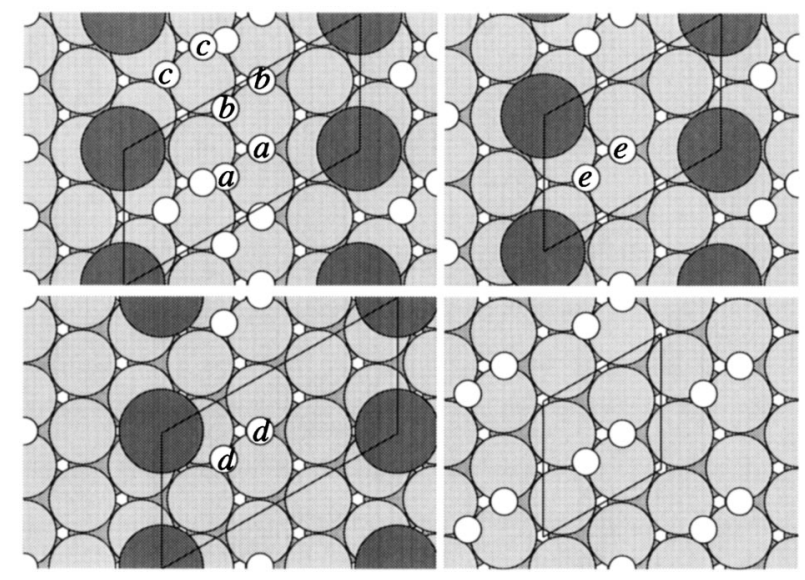

FIG. 2. Transition states for $\mathrm{N}_{2}$ dissociation in different configurations. Top left: $\mathrm{Na}$ covered surface in a $(2 \times 4)$ unit cell. Bottom left: Same as above except that $\mathrm{Na}$ is in a hcp site. Top right: Na covered surface in a $(2 \times 3)$ unit cell. Bottom right: $(2 \times 2)$ unit cell on a clean Ru surface. $a-a$, $b-b, c-c, d-d$, and $e-e$ indicate the position of the transition state $\mathrm{N}-\mathrm{N}$ molecule. 


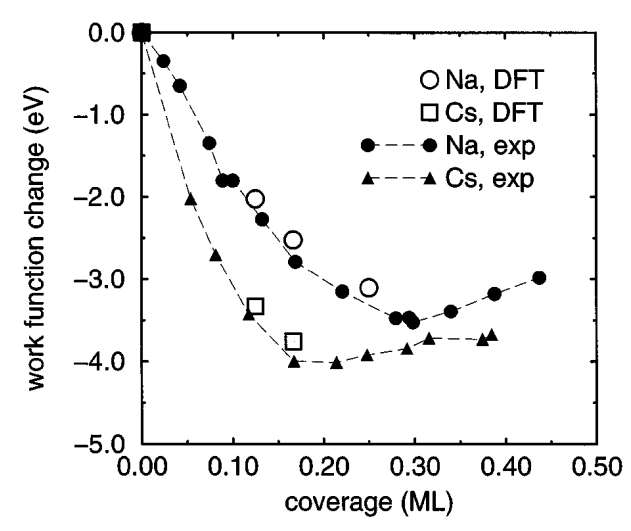

FIG. 3. Work function change induced by Na and Cs adsorption on $\mathrm{Ru}(0001)$ as a function of coverage. The open symbols are calculated results (circles and squares, respectively), and the filled symbols show experimental data from Ref. [20]. The work function for the clean surface is calculated to be $5.2 \mathrm{eV}$; the experimental value is $5.1 \mathrm{eV}$ [21].

different geometries tested are illustrated in Fig. 2, and in Fig. 4 we show $\Delta E_{\mathrm{TS}}$ for the different configurations. As discussed above, the effect of Cs is considerably stronger than of $\mathrm{Na}$, and it can be seen that $\left|\Delta E_{\mathrm{TS}}\right|$ increases as the alkali atoms get closer to the transition state complex (configurations $c$ and $d$ are lower in energy than $a$ and $b$ because they are closer to the alkalis, cf. Fig. 2).

In Fig. 4 , we have plotted $\Delta E_{\mathrm{TS}}$ as a function of the quantity

$$
\Delta E_{\mathrm{dip}}=-\varepsilon \mu,
$$

which is the electrostatic interaction between a dipole with electric dipole moment $\mu$ and an electric field $\varepsilon$. For $\mu$ in Eq. (3), we have simply taken the calculated dipole moment of the transition state complex in the absence of the alkali:

$$
\mu=\int d \vec{r} \Delta \rho_{\mathrm{TS}}(\vec{r}) z
$$

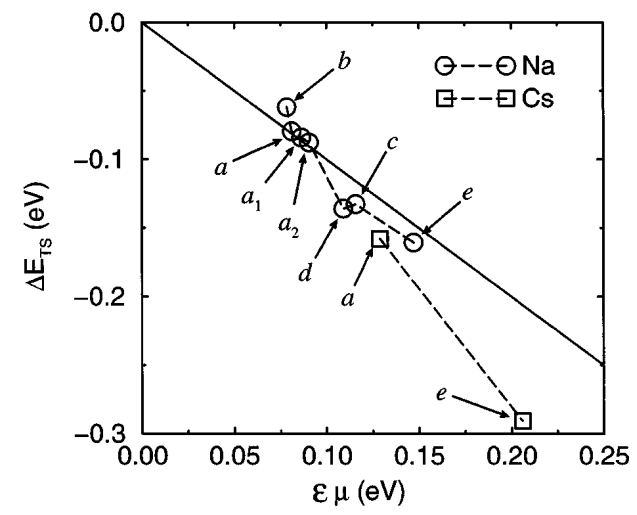

FIG. 4. Interaction energy between alkali atoms and $\mathrm{N}_{2}$ in the transition state for dissociation as a function of $\varepsilon \mu$. The alkali atoms are $\mathrm{Na}$ (circles) and $\mathrm{Cs}$ (squares). The configurations $a_{1}$ and $a_{2}$ correspond to an $a$ configuration, with the height of the $\mathrm{Na}$ atom increased from the equilibrium height by 0.1 and $0.2 \AA$, respectively (see Fig. 2). where $\Delta \rho_{\mathrm{TS}}=\rho_{\mathrm{TS}}^{\mathrm{N}_{2} / \mathrm{Ru}}-\rho^{\mathrm{Ru}}$ with the $\rho$ 's including electronic and ionic charge density contributions. The value we find is $\mu=-0.13 \mathrm{e} \AA$. The electric field $\varepsilon$ we determine from the alkali-induced electrostatic potential $\Delta \phi_{\text {alkali }}=\phi_{\text {alkali } / \mathrm{Ru}}-\phi_{\mathrm{Ru}}$ plotted along a line perpendicular to the surface through the center of mass of the adsorbate complex (see Fig. 5). We use the maximum field outside the surface as a measure of $\varepsilon$ rather than attempting to find the local field at the height of the adsorbate. For the present purposes, this can be taken as an empirical choice, but a more detailed analysis of the electrostatic interaction between adsorbates shows that the screening of the field in the adsorbate region should not be included, and the field just outside the surface is a reasonable measure of the nonscreened field $[10,22]$.

It is clear from Fig. 4 that there is a very good correlation between $\Delta E_{\mathrm{TS}}$ and $\Delta E_{\mathrm{dip}}$. At higher values of $\Delta E_{\mathrm{dip}}$, there is a tendency that $\Delta E_{\mathrm{TS}}$ varies even more strongly than $\Delta E_{\mathrm{dip}}$. This is what one would expect: For small fields the interaction is proportional to the field, while for larger fields polarization effects introduce an attractive term which is second order in the field strength [10]. The correlation in Fig. 4 strongly suggests that the direct electrostatic interaction between the adsorbed alkalis and the transition state complex dominates the promotion effect. We have also checked that there is no correlation between the alkali-induced change in adsorption energy and the change in the density of the states at the Fermi level. In fact, the latter changes extremely little as can be seen from the inset in Fig. 5.

We can also consider what determines the energy change for the adsorbed $\mathrm{N}_{2}$ molecule and for the dissociated state. In the molecularly adsorbed state, the $\mathrm{N}_{2}$ induced dipole moment is extremely small $(+0.01 \mathrm{e} \AA$ for the six layer slab and -0.01 e for the two layer slab). This is too little to give the effect seen in Fig. 1. For such small dipole moments, another effect, which we

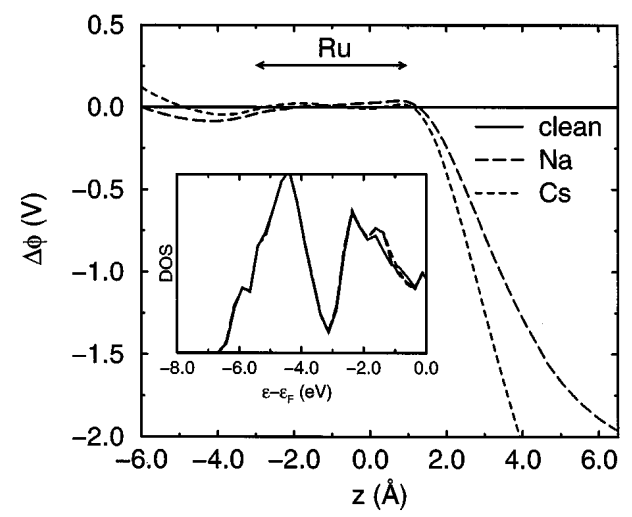

FIG. 5. Na- and Cs-induced electrostatic potential plotted along lines perpendicular to the surface through the fcc site farthest away from the alkali atoms (see Fig. 2, top left). The arrow shows the position of the slab (the $\mathrm{Ru}$ layers are at $z=0.0$ and $-2.2 \AA$ ). The inset shows the local density of states projected onto a $\mathrm{Ru}$ atom neighboring the dissociating molecule in configuration $c$. 
ascribe to a Pauli repulsion, dominates [22]. We note that, for molecular adsorbates with a larger dipole moment, the electrostatics still dominates. For $\mathrm{CO}$ adsorbed on $\mathrm{Ru}(0001)$, we calculate a much larger dipole moment of $-0.11 \mathrm{e} \AA$, and here the electrostatic interaction also describes the interaction with Na very well [22].

For the dissociated state, the dipole moment is $-0.10 \mathrm{e} \AA$. This is smaller than for the transition state, and the effect of Na adsorption [ $-0.07 \mathrm{eV}$ (see Fig. 1)] is correspondingly smaller. Again the interaction is dominated by electrostatics. According to our calculations, the alkali promotion thus provides a means of lowering the energy barrier while changing the stability of the reactants and the products considerably less. Alkali promotion of $\mathrm{N}_{2}$ therefore does not follow the Brøndsted-Polanyi relations [23], where the lowering of a reaction barrier is a fraction of the lowering of the potential energy of either the reactant or the product.

DFT calculations for $\mathrm{H}_{2}$ dissociation on $\mathrm{Pd}(100)$ have shown that a $(2 \times 2)$ overlayer of $\mathrm{K}$ and $\mathrm{S}$ increases the barrier $[11,24]$. It is concluded that no single contribution to the interaction energy can explain this behavior. Our results are simpler to interpret for three reasons: (i) The $\mathrm{N}_{2}$ transition state has a much larger dipole moment, (ii) $\mathrm{Na}$ and $\mathrm{Cs}$ do not induce any changes in the $d$ bands of the surrounding $\mathrm{Ru}$ atoms, and (iii) the unit cells used are larger (the adsorbates are not forced to be very close to each other). Point (i) means that the electrostatic interaction is stronger, and points (ii) and (iii) ensure that, at the same time, the direct or indirect interaction is weaker. We saw above that the dipole moment of molecularly adsorbed $\mathrm{N}_{2}$ is much smaller than for $\mathrm{N}_{2}$ in the transition state. For molecular $\mathrm{N}_{2}$, reason (i) is therefore less dominant, and the simple electrostatic picture of the alkali- $\mathrm{N}_{2}$ interaction does not hold. In general, there are thus several interaction mechanisms in coadsorption, but, for the interaction of adsorbed alkali atoms with the transition and final states of dissociating $\mathrm{N}_{2}$ on $\mathrm{Ru}$, the electrostatics dominates.

The variation of the field along the surface gives rise to forces acting on the transition state complex due to the alkali atoms. These forces will tend to change the minimum energy path so that in the presence of the alkali atoms the path and the transition state geometry will not be completely the same as the one determined from the clean surface calculation. The change in the transition state energy $\left|\Delta E_{\mathrm{TS}}\right|$ considered above is therefore a lower bound on the change in the true activation barrier. We have estimated the effect of including relaxation of the transition state from the alkali-induced forces on the transition state complex and the calculated curvature of the potential energy surface around the transition state on the clean surface. For the worst case, the correction amounts to $30 \mathrm{meV}$.

We conclude that DFT calculations are able to describe in some detail the promotional effect of alkali adsorbates on the dissociation of $\mathrm{N}_{2}$ and thus the promotion of ammonia synthesis on $\mathrm{Ru}$ catalyst surfaces. We also conclude that the main component of this effect is an electrostatic interaction between the adsorbed alkalis and the transition state complex.

The present work was, in part, financed by The Danish Research Councils through The Center for Surface Reactivity and Grant No. 9501775. The Center for Atomicscale Materials Physics is sponsored by the Danish National Research Foundation.

[1] A. Nielsen, Catal. Rev. 4, 1 (1970).

[2] S. R. Tennison, in Catalytic Ammonia Synthesis, edited by J. R. Jennings (Plenum, New York, 1991), p. 303.

[3] O. Hinrichsen, F. Rosowski, M. Muhler, and G. Ertl, Chem. Eng. Sci. 51, 1683 (1996).

[4] G. Ertl, S. B. Lee, and M. Weiss, Surf. Sci. 114, 527 (1982).

[5] S. Bare, D. R. Strongin, and G. A. Somorjai, J. Chem. Phys. 90, 4726 (1986); G. A. Somorjai and N. Materer, Top. Catal. 1, 215 (1994).

[6] K.-I. Aika, T. Takano, and S. Murata, J. Catal. 136, 126 (1992); A. Ozaki and K. Aika, in Catalysis: Science and Technology, edited by J. R. Anderson and M. Boudart (Springer-Verlag, Berlin, 1981), Vol. 1.

[7] For a review, see, e.g., Physics and Chemistry of Alkali Metal Adsorption, edited by H.P. Bonzel, A. M. Bradshaw, and G. Ertl (Elsevier, Amsterdam, 1989).

[8] R. A. de Paola, F. M. Hoffmann, D. Heskett, and E. W. Plummer, Phys. Rev. B 35, 4236 (1987).

[9] P. J. Fiebelman and D. R. Hamann, Surf. Sci. 149, 48 (1985).

[10] J. K. Nørskov, S. Holloway, and N.D. Lang, Surf. Sci. 137, 65 (1984); J. K. Nørskov, in Ref. [7], p. 253.

[11] S. Wilke and M. H. Cohen, Surf. Sci. 380, L446 (1997).

[12] H. Dietrich, P. Geng, K. Jacobi, and G. Ertl, J. Chem. Phys. 104, 375 (1996).

[13] L. Romm, G. Katz, R. Kosloff, and M. Asscher, J. Phys. Chem. B 101, 2213 (1997).

[14] J. P. Perdew et al., Phys. Rev. B 46, 6671 (1992).

[15] G. Kresse and J. Furthmüller, Comput. Mater. Sci. 6, 15 (1996).

[16] S. G. Louie, S. Froyen, and M. L. Cohen, Phys. Rev. B 26, 1738 (1982).

[17] A. Ulitsky and R. Elber, J. Chem. Phys. 92, 1510 (1990).

[18] J. J. Mortensen, Y. Morikawa, B. Hammer, and J. K. Nørskov, J. Catal. 169, 85 (1997).

[19] H. Over, H. Bludau, M. Skottke-Klein, G. Ertl, W. Moritz, and C. T. Campbell, Phys. Rev. B 45, 8638 (1992).

[20] G. Pirug, C. Ritke, and H.P. Bonzel, Surf. Sci. 257, 50 (1991).

[21] S. R. Kelemen and T.E. Fisher, Surf. Sci. 87, 53 (1979).

[22] J. J. Mortensen, B. Hammer, and J. K. Nørskov, Surf. Sci. (to be published).

[23] See, e.g., R. A. van Santen and M. Neurock, Catal. Rev. Sci. Eng. 37, 557 (1995); R. I. Masel, Principles of Adsorption and Reaction on Solid Surfaces (Wiley, New York, 1996).

[24] S. Wilke and M. Scheffler, Phys. Rev. Lett. 76, 3380 (1996). 